\title{
Difficulties in Breastfeeding: Easy Solution by Oketani Breast Massage
}

\author{
Tasnim $\mathrm{S}^{1}$, Roy $\mathrm{SK}^{2 *}$, Jahan $\mathrm{K}^{2}$, Nazmeen $\mathrm{S}^{3}$, Debnath $\mathrm{SC}^{2}$, Islam $\mathrm{ABMM}^{4}$ \\ ${ }^{I}$ Department of Obstetrics \& Gynaecology, Institute of Child and Mother Health, Dhaka, Bangladesh; \\ ${ }^{2}$ Bangladesh Breastfeeding Foundation, Dhaka, Bangladesh; ${ }^{3}$ Department of Obstetrics \& \\ Gynaecology, Centre for Women and Child Health, Dhaka, Bangladesh; ${ }^{4}$ Institute of Public Health \\ Nutrition, Dhaka, Bangladesh.
}

\begin{abstract}
Background: Breastfeeding is universally recommended feeding method for promoting child survival. Many mothers in developing countries do not practice optimum breastfeeding due to difficulties in the breast that may lead to temporary or permanent cessation of breastfeeding. Oketani breast massage approach is one such technique to overcome those difficulties for successful lactation to promote child growth. The aim of this study was to assess the difficulties in breastfeeding and mother's expression on benefits of Oketani breast massage.

Methods: A cross sectional study was conducted at lactational management center (LMC) in the Institute of Child Mother Health (ICMH), Dhaka, Bangladesh during March to August 2015. A total of 98 mothers (having 0-12 month's old children) who had breast problems or difficulty in breastfeeding were included in the study. The mothers agreed to undertake Oketani massage. This is a breast massage that promotes breast milk production and helps the lactating mothers to overcome certain difficulties in the breast. Mothers who received Oketani massage were interviewed using a semi-structured questionnaire.
\end{abstract}

Results: Ninety-eight mothers were interviewed for this study. About two-thirds (66.3\%) of babies were girls of which $57.1 \%$ were less than 2 months. More than two-thirds (78.6\%) mother ages were more than 25 years. Half of the mothers completed secondary school certificate (SSC) in which non-working (63.3\%) mothers were more than working mother (36.7\%). The proportion of exclusive breastfeeding practice was 50.0\%. Most common difficulties included breast engorgement (22.4\%), followed by sore nipple (17.3\%) and insufficient milk (14.3\%). After receiving Oketani massage mother's perception of getting confidence improved to $80.6 \%$. About $69 \%$ said that the baby can be fed well, $61.2 \%$ felt that there was increase in breast milk production and $56.1 \%$ mothers expressed feeling good after the massage. Study findings revealed that Breast feeding significantly improved with the Oketani breast massage $(\mathrm{p}=0.001)$.

Conclusion: Oketani massage is considered as a useful technique to build confidence and improved milk secretion among mothers facing difficulty during lactation. A multicentre study with detailed assessment of quality, the quantity of breastmilk as well as baby's growth and nutritional assessment is recommended.

Keywords: Breastfeeding; Oketani breast massage; Lactational problem; Mastitis

\section{Introduction}

Breastfeeding promotes sensory and cognitive development and protects the infant against infectious and chronic diseases in later life. Exclusive breastfeeding reduces infant mortality and quicker recovery during illness. ${ }^{1}$

It is also important for mothers as it delays the return of menstruation. Lack of exclusive. Breastfeeding during the first six months of life is considered as important risk factor for infant and childhood morbidity and mortality, poor school performance, reduced productivity, and impaired intellectual development. ${ }^{2-4}$ Early introduction of complementary foods increases infant morbidity

Correspondence: Dr. SK Roy, Bangladesh Breastfeeding Foundation (BBF), Mohakhali, Dhaka-1212, Bangladesh. e-mail: skroy1950@gmail.com; ORCID: 0000-0001-7750-9831 and mortality by reducing the protective factors present in breast milk and increasing the exposure to external contamination. ${ }^{4}$

Mother's intention to breastfeed the babies are usual but sometimes problems may interfere optimum infant feeding.5Common problems that may lead to the temporary or permanent cessation of breastfeeding include engorgement, plugged ducts and mastitis. ${ }^{6}$

According to the World Health Organization, although engorgement, plugged ducts and mastitis are distinct entities, their pathogenesis involves the common problem of stasis of breast milk. ${ }^{7}$ A few mothers may be unable to overcome the problems of breastfeeding with conventional solutions with counseling and demonstration of position and attachment. ${ }^{6}$ Newer techniques like Oketani breast 
massage can be of great help for those mothers. The Oketani Lactation Management is a unique breast management created by Ms. Sotomi Oketani of Japan.8Sotomi also theorized that breastfeeding enhances mother and child bonding while also encircling the mother and child's physical and mental condition by natural means. ${ }^{8-9}$

Study from Bangladesh shows overall 30-37\% percent mothers with lactation problem need help with Oketani technique to sustain exclusive breastfeeding. ${ }^{10-11}$ Although this has been practiced in Bangladesh since 1994 with the great challenges of the scarcity of trained personnel. ${ }^{11}$ Japanese midwives provide short training of 4-5 days from time to time in a group of 12-15 nurse/ midwives of different hospitals which were coordinated by Institute of Child Mother Health (ICMH). However, there was lack of monitoring and gaps in creating demand among the hospital authorities to support this service and as such the technique could not be made available to the wider population. ${ }^{8}$ Till date,Oketani technique is available in a few selected centres in Bangladesh. ${ }^{11-12}$

The perception of mothers about Oketani breast massage is yet not studied in Bangladesh. The objective of the present study was to find out the difficulties in breastfeeding and mother's perception after getting Oketani breast massage.

\section{Materials and Methods}

Study design and settings: A crosssectional study was conducted in lactation management center (LMC) of Institute of Child \& Mother Health (ICMH). The center is located near the capital city of Dhaka, Bangladesh. The study was conducted from March to August 2015.

Study population and sampling: All mothers who have 0-12 month's old children attending at LMC with difficulty in breastfeeding were included in the study. A total 98 mothers were recruited in the study by simple random sampling.

Oketani breast massage technique: Oketani calls the part between the breast and the base of the underlying chest muscle. Oketani breast massage is special types of manual technique of breast treatment developed by Sotomi Oketani to promote breastfeeding especially in certain lactation problem like not enough milk or sore nipple or mastitis. ${ }^{8}$ This is a connective tissue massage without pain and that stimulates the mother, loosen the tissues and promotion of secretion, so that baby can suck easily. There are 8 different techniques in the manual massage of the breast which is numbered as $1,2,3$,
4, 5, 6, 7 and 8. Massages 1 to 7 are called one set or course of treatment and it is different in both breasts.
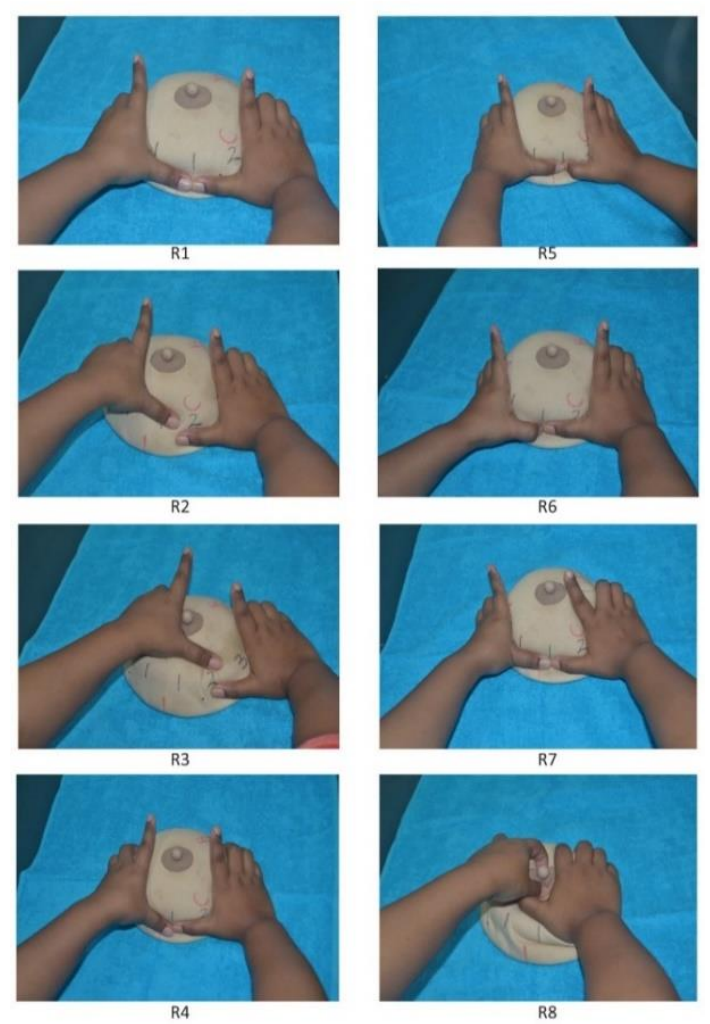

Figure I: Oketani massage technique for right breast

One set of massage and expression are completed within one minute and this is repeated for 15-20 minutes. ${ }^{9}$

Data collection: A semi-structured pretested questionnaire was used for collection of data. Data were collected on feeding problem, age, (both of mother and baby), gender and place of delivery. During the assessment, breastfeeding was observed for identification of problems. Breastfeeding observation form was used as a guide for assessment. Mothers were categorized for the requirement of services according to their need like counseling, correction of position and attachment as well as Oketani breast massage technique. After observation of breastfeeding, all the mothers were counselled every day by the trained nurses. After demonstration with the help of models about proper position and attachment mothers were asked to breastfeed the babies applying proper technique. Nurses and midwives helped the mothers if any of them was unable to adopt the proper technique.

Data analysis: All data were checked manually for completeness and consistency. Data were entered and analyzed using SPSS version 20. Descriptive 
statistics included mean and standard deviation of continuous variables and proportions for categorical variables. Chi-squared test was used if the variables were categorical.

Ethical issues: The study was approved by the Ethical Review Committee (ERC) of Institute of Child Mother Health (ICMH). Both verbal and written consent were taken from participants. Privacy and confidentiality were maintained throughout the study period by personal identifiers during data collection.

\section{Results}

A total of 98 mothers attended the lactation management center. About two-thirds (66.3\%) of the children were girls and rest of them were boys. More than half of children's (57.1\%) age were less than 2 months and $15.3 \%$ children's age were between 2 to 3 months. The age distribution of mothers shows that more than two-thirds (78.6\%) of mothers were aged less than 25 years and rest of them $(21.4 \%)$ were more than 25 years of age. Regarding the educational status of mothers, half $(50.0 \%)$ of the mothers completed secondary school certificate (SSC) and a considerable portion (7.1\%) had no education. Non-working mothers were in higher proportion than working mothers (63.3\% vs 36.7\%). Caesarian delivery were more than normal delivery $(64.3 \%)$ vs $(35.7 \%)$.

The pattern of feeding practices of children according to their age also assessed (table II). The prevalence of exclusive breastfeeding rate $33.7 \%$, $9.2 \%$ and $7.1 \%$ among infants were $<2$ months, $2-3$ months and 4-6 months respectively.

Table I: General characteristics of mothers and children

\begin{tabular}{llc}
\hline \multicolumn{1}{c}{ Characteristics } & n & \% \\
\hline Gender of the Child & & \\
Boys & 33 & 33.7 \\
Girls & 65 & 66.3 \\
Age of the Child & & \\
< 2 months & 56 & 57.1 \\
2-3 months & 15 & 15.3 \\
$4-6$ months & 12 & 12.3 \\
$>$ 6 months & 15 & 15.3 \\
Age of mother & & \\
$\leq 25$ years & 21 & 21.4 \\
$>$ 25 years & 77 & 78.6 \\
Education of mother & & \\
Illiterate & 07 & 7.1 \\
Primary & 19 & 19.4 \\
Secondary & 23 & 23.5 \\
SSC and above & 49 & 50.0 \\
Occupation of mother & & \\
Working & 36 & 36.7 \\
Non-working/housewives & 62 & 63.3 \\
Mode of Delivery & & \\
Normal & 35 & 35.7 \\
Caesarean & 63 & 64.3 \\
\hline
\end{tabular}

On the other hand, breast milk with formula was given to $15.3 \%, 3.1 \%, 8.2 \%$ and $2.0 \%$ among $<2$ months, 2-3 months and 4-6 months, >6 months children, respectively. However, only $13.2 \%$ children were given breast milk with family foods like khichuri, suji etc.

Table II: Current feeding practices of children according to children's age

\begin{tabular}{|c|c|c|c|c|c|c|c|c|}
\hline \multirow{3}{*}{ Characteristics } & \multicolumn{8}{|c|}{ Age of children } \\
\hline & \multicolumn{2}{|c|}{$\begin{array}{l}<2 \\
\text { months }\end{array}$} & \multicolumn{2}{|c|}{$\begin{array}{c}2-3 \\
\text { months }\end{array}$} & \multicolumn{2}{|c|}{$\begin{array}{c}\text { 4-6 } \\
\text { month } \\
\text { s }\end{array}$} & \multicolumn{2}{|c|}{$\begin{array}{c}>6 \\
\text { months }\end{array}$} \\
\hline & $\mathrm{n}$ & $\%$ & $\mathrm{~N}$ & $\%$ & $\mathrm{n}$ & $\%$ & $\mathrm{n}$ & $\%$ \\
\hline $\begin{array}{l}\text { Breast milk } \\
\text { only } \\
\text { (Exclusive } \\
\text { Breast } \\
\text { Feeding) }\end{array}$ & $\begin{array}{l}3 \\
3\end{array}$ & $\begin{array}{l}33 . \\
7\end{array}$ & 9 & $\begin{array}{l}9 . \\
2\end{array}$ & 7 & $\begin{array}{l}7 . \\
1\end{array}$ & 2 & 2.0 \\
\hline $\begin{array}{l}\text { Breast milk } \\
\text { with } \\
\text { formula } \\
\text { milk }\end{array}$ & $\begin{array}{l}1 \\
5\end{array}$ & $\begin{array}{l}15 . \\
3\end{array}$ & 3 & $\begin{array}{l}3 . \\
1\end{array}$ & 8 & $\begin{array}{l}8 . \\
2\end{array}$ & 2 & 2.0 \\
\hline $\begin{array}{l}\text { Breast milk } \\
\text { with family } \\
\text { food like } \\
\text { khichuri, } \\
\text { suji etc. }\end{array}$ & 1 & 1.1 & 0 & $\begin{array}{l}0 . \\
0\end{array}$ & 5 & $\begin{array}{l}5 . \\
1\end{array}$ & $\begin{array}{l}1 \\
3\end{array}$ & $\begin{array}{l}13 . \\
2\end{array}$ \\
\hline
\end{tabular}

Breast problems were assessed by lactation experts and different breast problems were identified. It was found that about $22.4 \%$ mothers suffered from breast engorgement followed by sore nipple $(17.3 \%)$, insufficient milk in $14.3 \%, 9.2 \%$ had block ducted, $6.1 \%$ had inverted nipple, $3.0 \%$ had flat nipple and $2.0 \%$ mothers had poor attachment (figure 2).

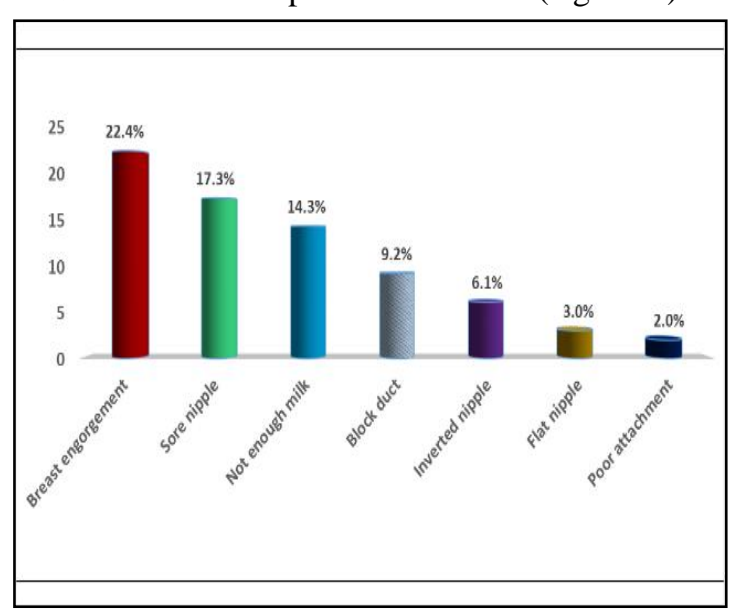

Figure 2: Distribution of difficulties faced by the mother (multiple responses)

Mother's perception after receiving Oketani massage was studied (table III). Maximum $(80.6 \%)$ of the mothers grew confidence, $69.4 \%$ stated that baby could feed well, $61.2 \%$ felt there was increase in Breastmilk production and $56.1 \%$ mothers expressed feeling good after massage. 
To get satisfactory feeding, the majority of them $(41.0 \%)$ required massage for $2-3$ times, $(27.0 \%)$ needed to cure single and $25 \%$ needed three times massages. Only 2.0\% mothers needed Oketani massage for 5 times for gaining satisfaction (figure 3).

Table III: Mother's perception after receiving Oketani massage

\begin{tabular}{lcc}
\hline \multicolumn{1}{c}{ Perception features } & n & \% \\
\hline Feeling good & 55 & 56.1 \\
Soften breast & 40 & 40.8 \\
Increase breast milk & 60 & 61.2 \\
secretion & & \\
Complete emptying of breast & 27 & 27.6 \\
Baby can be feed well & 68 & 69.4 \\
Decrease breast pain & 51 & 52 \\
Resolved nipple problem & 22 & 22.4 \\
Feel confident & 79 & 80.6 \\
\hline
\end{tabular}

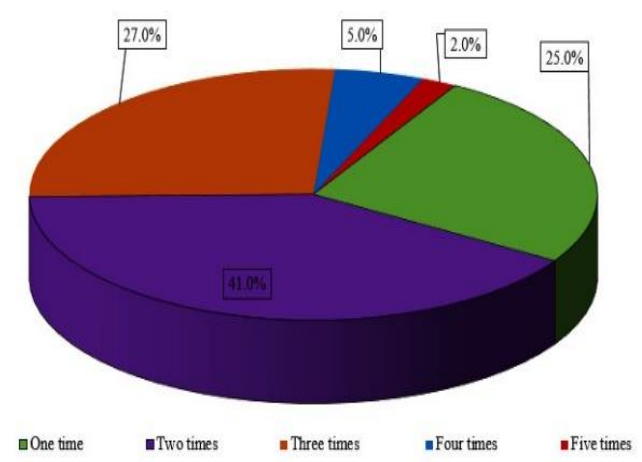

Figure 3: Distribution of the frequency of the Oketani massage to obtain satisfaction.

It was revealed that only "baby can be feed well" was significantly associated with the frequency of Oketani breast massage $(p=0.001)$ (table IV).

Table IV: Relation between mother's perception and frequency of Oketani breast massage

\begin{tabular}{lcccccc}
\hline \multirow{2}{*}{ Characteristics } & \multicolumn{3}{c}{ Frequency of Oketani breast massage } & & P value \\
\cline { 2 - 6 } & One & Two & Three & Four & Five & $\mathrm{n}(\%)$ \\
\hline Feeling good & $\mathrm{n}(\%)$ & $\mathrm{n}(\%)$ & $\mathrm{n}(\%)$ & $\mathrm{n}(\%)$ & $\mathrm{n}(\%)$ & 0.752 \\
Soften breast & $9(3.06)$ & $27(67.5)$ & $8(69.2)$ & $1(20.0)$ & $0(0.0)$ & 0.269 \\
Increase breast milk secretion & $3(12.0)$ & $23(57.5)$ & $13(50.0)$ & $1(20.0)$ & $0(0.0)$ & 0.095 \\
Full empty of breast & $12(48.0)$ & $24(60)$ & $19(73.1)$ & $4(80.0)$ & $1(50.0)$ & 0.095 \\
Baby feed well & $5(20.0)$ & $13(32.5)$ & $8(30.8)$ & $1(20.0)$ & $0(0.0)$ & 0.939 \\
Decrease breast pain & $9(36.0)$ & $32(80.0)$ & $21(80.1)$ & $5(100.0)$ & $1(50.0)$ & 0.002 \\
Resolved nipple problem & $9(36.0)$ & $26(65.0)$ & $14(53.8)$ & $2(40.0)$ & $0(0.0)$ & 0.974 \\
Feel confidence during breastfeeding & $21(84.0)$ & $29(72.5)$ & $24(92.3)$ & $4(80.0)$ & $1(50.0)$ & 0.936 \\
\hline
\end{tabular}

\section{Discussion}

Breast massage is widely used throughout the world as a treatment for breastfeeding problems. There are many techniques used for breast massage that is documented, including the Oketani Lactation Management. The Oketani technique was introduced by Japanese midwife Sotomi Oketani in 1940 in Japan during the 2nd world war. ${ }^{9}$

In the present study, most of the mothers (78.6\%) were more than 25 years. Whereas a cross sectional study at LMC in Bangladesh found that most of the mothers $(55.0 \%)$ were in the age group 15-20 years. There is a difference with the present study findings.
This difference may due to different cultural and geographical variations of the study sample.

In the present study, most of the mothers were nonworking/housewives, whereas in a study by Februhartanty et al where $87.4 \%$ mothers were the working mother, which differ to our finding. ${ }^{12}$ These differences may be due to the different sociodemographic status of the mothers.

A cross-sectional study conducted by Hossain et al in a lactational management center of Bangladesh on problems during lactation included breast engorgement, nipple sore, cracked nipple, flat nipple and poor milk production. ${ }^{13} \mathrm{~A}$ review by Giugliani, 
2004 on problems during lactation included breast engorgement, nipple pain/trauma, plugged milk duct, breast infection and poor milk production. ${ }^{14}$

In the current study, the first problem was breast engorgement, the second problem was sore nipple and the third one was not enough milk production. A cross sectional study in urban Nepal reported that the main reason for introducing other foods before six months of age was perceived insufficient breast milk. ${ }^{15} \mathrm{~A}$ cross-sectional study in Indonesia reported that about one-third had poor milk production that was their first problem and the second problem was sore nipple. ${ }^{12}$ These findings are inconsistent with the present study findings in which mothers had sore nipple. In a study by Februhartanty et al the first two most common problems encountered during lactation were related to emotional state and tiredness, but in our study, emotional and physical support for the mothers were not explored. ${ }^{12}$ It is reported that Oketani breast massage stimulates the physical and mental development of infants improving both their mood and sleep pattern by raising the quality of breast milk. ${ }^{9}$

In this study, the improper attachment was noticed only about $2.0 \%$ and those mothers were shown the correct technique of breastfeeding by nurses. When the mothers were given Oketani massage for the increase of breast milk, it provided great satisfaction and enthusiasm for the mothers. ${ }^{16}$ In the present study, Oketani massage was applied to treat the breast problems in which the maximum mother's felt confident and they stated that now their babies were fed well and had increased secretion of breast milk (61.2\%). A cross-sectional study at LMC of Rajshahi Medical College Hospital, Bangladesh and the study findings revealed that maximum mothers were practically helped by showing correct position and attachment of the baby to the breast. ${ }^{13}$ The present study findings are similar to that of Thomsen et al who found symptomatic improvement of mastitis with emptying of the breast. ${ }^{17}$

Although Oketani technique is being used in Bangladesh for relatively long time, studies the pattern of problems and their solution through the application of Oketani massage has not reported yet. In this study mother's perception about Oketani massage was positive and the majority of the mothers expressed that they feel confident and they stated that their babies were able to fed better than before. The present study also analysed the frequency of breast massage in which maximum mothers stated that 2 an 3 times massage even a single massage was sufficient to build confidence in some cases.

\section{Conclusion}

In the present study, lactation problems mostly found are breast engorgement and sore nipple that hampers to sustain exclusive breastfeeding. Oketani massage is considered to solve these problems by improving milk secretion among lactating mothers. who are facing difficulty with lactation. A multicentre study with detailed assessment of quality, the quantity of breastmilk as well as growth and nutritional assessment of children are recommended.

Conflict of Interest: All of the authors declare that no one has any conflict of interest with the research, authorship and or publication of this article.

\section{References}

1. WHO. Global strategy for infant and young child feeding: the optimal duration of exclusive breastfeeding. Geneva: WHO. 2001.

2. Institute of Health Metrics and Evaluation. Global burden of diseases, injuries, and risk factors study. Seattle, Washington USA. 2010.

3. Hall J. Effective community-based interventions to improve exclusive breastfeeding at four to six months in low- and low-middle-income countries: a systematic review of randomized controlled trials. Midwifery. 2011; 27:497-502.

4. World Health Organization. Guiding principles for complementary feeding of the breastfed child. Division of Health Promotion and Protection. Geneva; 2003.

5. Dewey KG, Cohen RJ, Brown KH, Landa Rivera L. Effects of exclusive breastfeeding for 4 versus 6 months on maternal nutritional status and infant motor development: results of two randomized trials in Honduras. J Nutr. 2001; 131:262-67.

6. Mangesi L, Dowswell T. Treatments for breast engorgement during lactation. Cochrane Database Syst Rev. 2010.

7. World Health Organization. Mastitis: Causes and Management. Geneva, Switzerland: World Health Organization; 2000.

8. Manual of Oketani Breast Massage Technique, Akaeda Medical Research Foundation, Tokyo, Japan. 1996. 
9. Oketani lactation management Japanese breast massage manual. 2004. Oketani lactation management association. Japan; 2004.

10. Kabir N, Tasnim S. Oketani lactation management: a new method to augment breast milk. J Bangladesh Coll Phys Surg. 2009; 27:155-59.

11. Akter S, Tasnim S, Bhuiyan MMA, Hasan A. A Study on post-partum breast problems of mothers attending at lactation management center (LMC). Bangladesh Med J. 2015; 44:136-39.

12. Februhartanty J, Bardosono S, Septiari AM. Problems during Lactation are associated with exclusive breastfeeding in DKI Jakarta province: Fathers potential roles in helping to manage these problems. Mal J Nutr. 2006; 12:167-80.

13. Hossain MA, Haque MI, Siddiqui AB, Bari MI. Activities of Lactation Management Centre of Rajshahi. The Journal of Teachers Association RMC 2005. 18:2.
14. Giugliani ER. Common problems during lactation and their management. Pediatric. 2004; 80:147-52.

15. Ulak M, Chandyo R, Mellander L, Shrestha P, Strand T. Infant feeding practices in Bhaktapur, Nepal: a cross-sectional, health facility based survey. Int Breastfeeding J. 2014; 7:1.

16. Oketani S. The Real Report of the Oketani's Manual technique of Breast Treatment. The Practice. 1992; 14.

17. Thomsen AC, Espersen T, Maigaard S. Course and treatment of milk stasis, noninfectious inflammation of the breast and infectious mastitis in nursing women. Am J Obstet Gynecol 1984; 149:492-95. 